\title{
Bottom- up Approach for Salient Region Detection using Fixed Patch Sized Segmentation
}

\author{
Ankita V. Raut \\ P.G. Student \\ Department of Computer Engineering \\ Late G. N. Sapkal College of Engineering
}

\author{
J. V. Shinde \\ Assistant Professor \\ Department of Computer Engineering \\ Late G. N. Sapkal College of Engineering
}

\begin{abstract}
Salient region detection refers to extracting important information from image while negotiating the remaining things. It can be used in many fields such as for compression of image by blurring unwanted part of image, classification of image, segmentation of object, recognition of object and many more. In this work, two different visual cues are combined together to overcome disadvantages of separate methods. In this method first image is segmented using segmentation algorithm and segmented image is given as input to two different visual cues that are compactness and local contrast and then both the maps are evaluated and combined together to obtain final saliency map.
\end{abstract}

\section{Keywords}

Contrast, Diffusion process, Compactness, Salient region, Segmentation.

\section{INTRODUCTION}

Visual Saliency is the quality which separates out image pixel, object or person in the image from the rest part of image which attracts attention of human. Generally in the image human's vision fixes on different points, that fixation depends different features of image such as color contrast, compactness of the image pixels, spread of background in the image as well as center of the image. Saliency region detection[1] is the technique which uses these different visual cues to highlight image features like shape, texture and boundary of the image.

Saliency region detection methods mainly fall into two categories stimulus driven and task driven and are also called as bottom-up [2-4] method and top-down[5] method respectively. In top-down method trained objects are used to identify salient object and in case of bottom-up approach different visual cues are considered such as compactness, local contrast, global contrast, uniqueness as well as background of the image to identify salient object.

These different methods had certain advantages and disadvantages when they are implemented individually such as in case of contrast based methods only color contrast of the image is taken into consideration for identifying salient object but this method fails when color of the background and foreground is similar to each other. In case of compactness based methods spatial variance of the pixels are considered to define salient object that is generally pixels having less spatial variance are the part of salient object and remaining pixels will define background. But, for the image in which salient object have large spread this method fails. Background based methods emphasis more on background instead of foreground to detect salient object. But when these methods are combined together then it provides better result than the individual methods. Thus in this work local contrast and compactness methods are combined together to form final saliency map.

\section{LITERATURE SURVEY}

Achanta et al.[6] provided local contrast based model to determine salient object by using color and luminance features of the image. In this method saliency region is calculated by comparing local contrast of the pixels with its surrounding pixels at different intensity levels. It calculates distance between average feature vector of inner region of image and outer region of image at different scales. Maps created for color and luminance in this method considers one vector value for all these maps. And in this method both original and generated maps have same resolution but as in case of contrast focus will be only on color so this method fails when foreground and background have similar colors.

Goferman et al.[7] provides global contrast based context aware saliency detection model in which it considers four principles to detect salient region and those principles are local features such as color as well as contrast, global features that deviates from the redundant features, visual features and lastly high level knowledge like human faces using face detection algorithm. So, in this method as features are combined together with the context of objects so it provides proper results. But it works well only for the objects whose context is given

Perrazi et al.[8] introduce compactness based model in which it calculates element uniqueness and distribution and combines these features for saliency detection. In this method input image is abstracted and divided into number of superpixels using segmentation algorithm and then uniqueness of the object can be found by finding infrequent object which stands out from surrounding objects or superpixels and distribution of elements can be highlighted by considering compactness of the superpixels from the surrounding region generally it has been observed that background have more spread over image than foreground. But this method fails when foreground have more spread over image compared to background.

Li Zhou et al.[9] proposed a background based model with manifold ranking in which instead of focusing more on foreground it focuses on background. In this method background is divided into four regions top, bottom, left and right and then distance between the pixels are calculated. This method considers the fact that distance between background pixel and more similar background region will be less than distance between background and foreground region. But for more complicated images this method fails.

\section{PROPOSED SYSTEM}

In proposed system two methods compactness and local contrast are combined together to overcome disadvantages of 
individual one. So proposed system contains mainly five stages:

1. Image Segmentation

2. Generation of compactness based saliency map

3. Generation of local contrast based saliency map

4. Evaluation of saliency values of both saliency maps

5. Final Integration

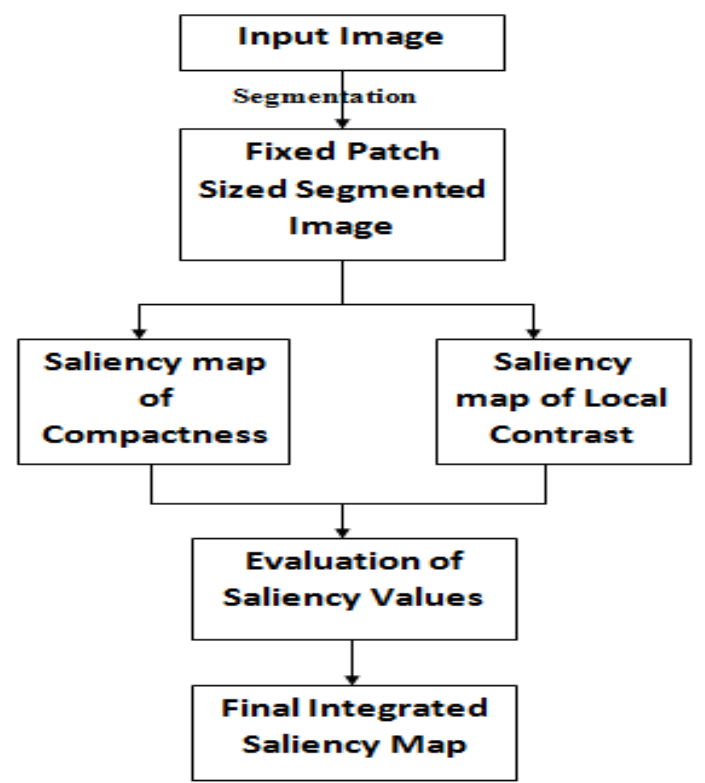

Fig 1: Proposed System Architecture

\section{Image Segmentation}

In this step, original image is segmented into number of superpixels by using segmentation algorithm.

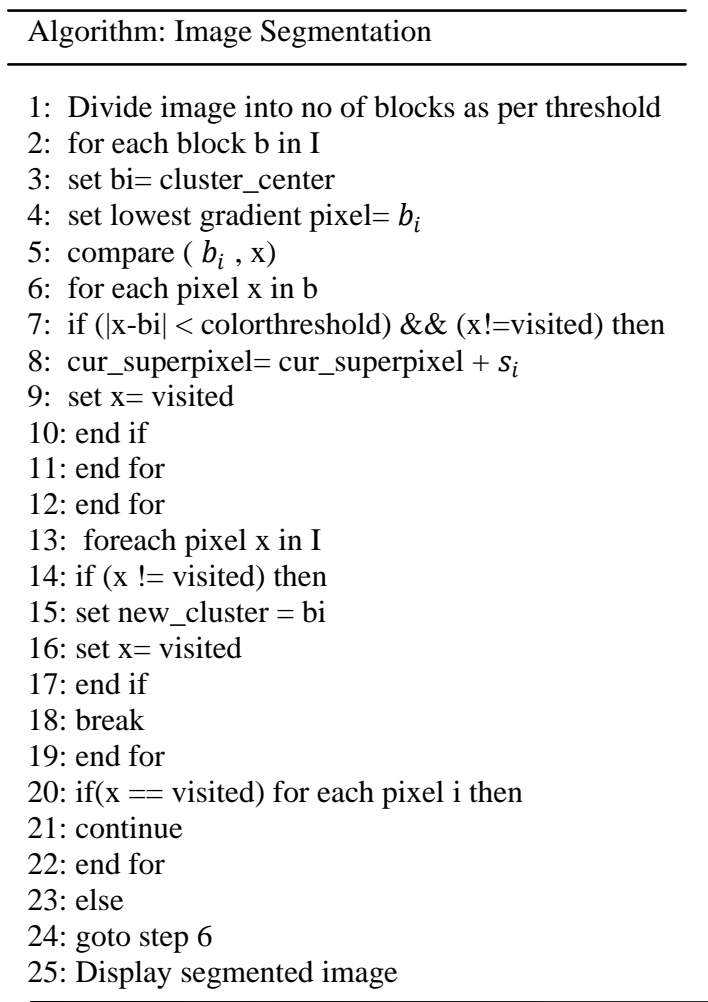

\section{Generation of compactness based saliency map}

Salient map of compactness is obtained by calculating distribution variance. Generally it is observed that human focuses at the center of image as well as foreground of the image have more compact distribution of pixels while at the background pixels have more spread. So considering these two facts distribution variance can be calculated by using following formula

$\operatorname{dv}(\mathrm{p})=\frac{\sum_{q=1}^{N} m_{p q} \cdot s_{q}\left\|c_{q}-i\right\|}{\sum_{q=1}^{N} m_{p q} \cdot s_{q}}$

Here, $m_{p q}=$ Value of similarity between superpixels $\mathrm{p}$ and $\mathrm{q}$.

$s_{q}=$ Total number of pixels in superpixel.

$c_{q}=$ Centroid of superpixel.

$\mathrm{i}=$ Center of the image

Distance variance for all superpixels gives saliency map of compactness $M_{\text {com }}$.

\section{Generation of local contrast based saliency map}

In this step color contrast is obtained by calculating color contrast variance between neighboring superpixels and salient object is identified by considering fact that generally salient object had less variance of color contrast so all the superpixels having less variance are combined together to obtain saliency map of local contrast. Color variance for superpixels can be calculated as

$\operatorname{cv}(\mathrm{p})=\frac{\sum_{q=1}^{N} v_{p q} \cdot s_{q}\left\|c_{q}-\mu t\right\|}{\sum_{q=1}^{N} v_{p q} \cdot s_{q}}$

Here, $v_{p q}=$ Value of similarity between superpixels $\mathrm{p}$ and $\mathrm{q}$.

$s_{q}=$ Total number of pixels in superpixel.

$c_{q}=$ Centroid of superpixel.

$\mu \mathrm{t}=$ Centroid of $M_{\text {com }}$.

Color contrast variance is calculated patch wise for each superpixel and saliency map $M_{\text {lcont }}$ for local contrast is obtained.

4. Evaluation of saliency values of both saliency maps After obtaining saliency maps of compactness and local contrast evaluation of their saliency values will be performed to get sharp edges which perfectly identifies salient object from the given input image.

\begin{tabular}{l} 
Algorithm: Evaluation of map \\
\hline Input: $M_{\text {com }}, M_{\text {lcont }}$ \\
Output: Saliency map \\
1: for each pixel i in superpixel \\
2: if $\left(M_{\text {com }}(i) !=M_{\text {lcont }}(i)\right)$ then \\
3: salient $=$ Evaluate $\left(M_{\text {com }}(i), M_{\text {lcont }}(i)\right)$ \\
4: else \\
5: salient (i) $=M_{\text {com }}(i)$ \\
6: end for \\
7: Function Evaluate $\left(M_{\text {com }}, M_{\text {lcont }}\right)$ \\
8: int difference $=\left|M_{\text {com }}-M_{\text {lcont }}\right|$ \\
9: if $($ difference $>$ threshold $)$ then \\
10: return difference \\
11: else \\
12: return $M_{\text {lcont }}$
\end{tabular}




\section{Final Integration}

After performing evaluation of these values which will improve sharpness of salient object both the maps are combined together to get final saliency map.

Final Saliency map $=M_{\text {lcont }}+M_{\text {com }}$

\section{RESULT \& ANALYSIS}

\section{A. Dataset}

Two datasets CSSD and ECSSD[10] are used to perform evaluation of results which contain near about 1000 images.

\section{B. Evaluation metric}

Correctness of salient region detection is performed by calculating precision and recall and comparing its results with the ground truth which is provided in dataset.

Precision: It is nothing but percentage of salient pixels that are correctly assigned to salient object with respect to ground truth mask that is ratio of true positive to true positive plus false positive.

Recall: It is percentage of salient pixels that are incorrectly assigned that is ratio of true positive to true positive plus false negative.

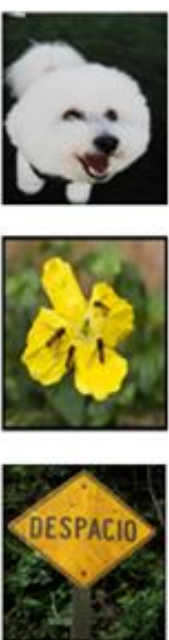

(a)
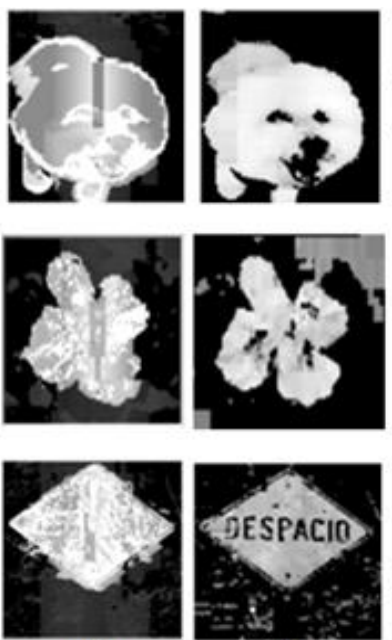

(b)
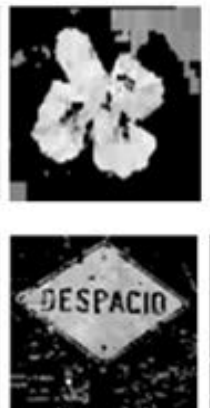

(c)
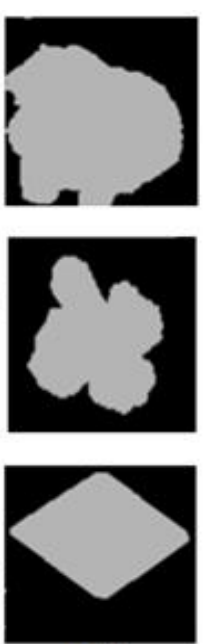

(d)
Fig 2: Saliency maps. (a) Original images. (b) Saliency map of compactness. (c) Saliency map of local contrast. (d) Final integrated result

Precision recall curve for comparing existing and proposed system can be given as follows

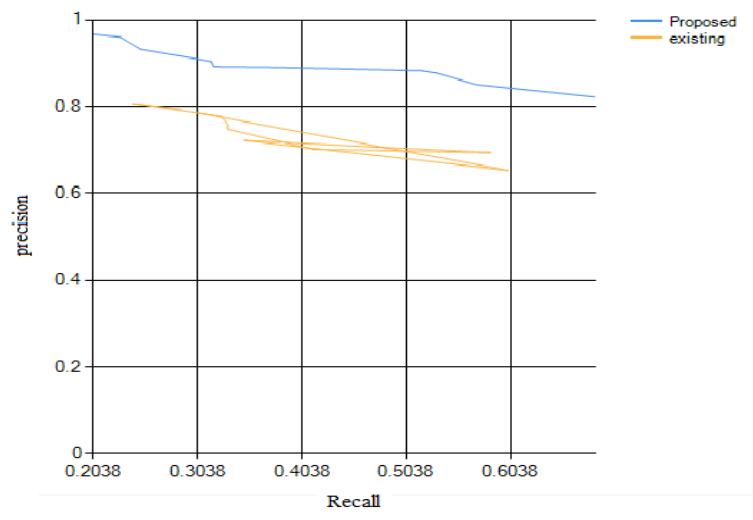

Fig 3: Precision Recall curve

\section{CONCLUSION \& FUTURE SCOPE}

In proposed system bottom up approach has been followed in which two important cues compactness and local contrast are combined together. In this method image is segmented into fixed sized patches and two different cues are combined together in which compactness identifies salient object and region that are suppressed by compactness will be highlighted by local contrast and finally evaluation on values obtained by these two methods will sharpen the edges of salient object so even in the case, when color of foreground and background is same proposed system performs better.

In future, context aware saliency can be used to predict salient object as well as some more features of image such as shape of object can be used to obtain more accurate results.

\section{REFERENCES}

[1] Li Zhou, Member, IEEE, Zhaohui Yang, and Ge Chang, "Salient Region Detection via Integrating DiffusionBased Compactness and Local Contrast," IEEE Trans. On Image Processing, Vol. 24, No. 11, November 2015.

[2] L. Itti, C. Koch, and E. Niebur, "A model of saliencybased visual attention for rapid scene analysis," IEEE Trans. Pattern Anal. Mach.Intell., vol. 20, no. 11, pp. 1254-1259, Nov. 1998.

[3] J. Harel, C. Koch, and P. Perona, "Graph-based visual saliency," in Proc. Adv. Neural Inf. Process. Syst., 2006, pp. 545-552.

[4] R. Achanta, S. Hemami, F. Estrada, and S. Süsstrunk, "Frequency-tuned salient region detection," in Proc. IEEE Conf. Comput. Vis. Pattern Recognit., Jun. 2009, pp. 1597-1604.

[5] C. Kanan, M. H. Tong, L. Zhang, and G. W. Cottrell, "SUN: Top-down saliency using natural statistics," Vis. Cognit., vol. 17, nos. 6-7, pp. 979-1003, 2009.

[6] R. Achanta, F. J. Estrada, P. Wils, and S. Süsstrunk, "Salient region detection and segmentation," in Proc. 6th ICVS, 2008, pp. 66-75.

[7] S. Goferman, L. Zelnik-Manor, and A. Tal, "Contextaware saliency detection," in Proc. IEEE Conf. Comput. Vis. Pattern Recognit. Jun. 2010, pp. 2376-2383.

[8] F. Perazzi, P. Krahenbuhl, Y. Pritch, and A. Hornung, "Saliency filters: Contrast based filtering for salient region detection," in Proc. IEEE Conf. Comput. Vis. Pattern Recognit., Jun. 2012, pp. 733-740.

[9] Li Zhou, Zhaohui Yang, and Ge Chang, "Salient Region Detection based on Compactness with Manifold Ranking," in 5th International Conference on Information Science and Technology, April 24-26 2015.

[10] Q. Yan, L. Xu, J. Shi, and J. Jia, "Hierarchical saliency detection," in Proc. IEEE Conf. Comput. Vis. Pattern Recognit., Jun. 2013, pp. 1155-1162.

[11] M.-M. Cheng, G.-X. Zhang, N. J. Mitra, X. Huang, and S.-M. Hu, "Global contrast based salient region detection," in Proc. IEEE Conf. Comput. Vis. Pattern Recognit., Jun. 2011, pp. 409-416.

[12] Y. Wei, F. Wen, W. Zhu, and J. Sun, "Geodesic saliency using background priors," in Proc. 12th Eur. Conf. Comput. Vis., 2012, pp. 29-42. 
[13] C. Yang, L. Zhang, H. Lu, X. Ruan, and M.-H. Yang, "Saliency detection via graph-based manifold ranking," in Proc. IEEE Conf. Comput. Vis. Pattern Recognit., Jun. 2013, pp. 3166-3173.

[14] Y. Zhai and M. Shah, "Visual attention detection in video sequences using spatiotemporal cues," in Proc. 14th Annu. ACM Int. Conf. Multimedia, 2006, pp. 815824.

[15] A. Borji, D. N. Sihite, and L. Itti, "Salient object detection: A benchmark," in Proc. 12th Eur. Conf. Comput. Vis., 2012, pp. 414-429.

[16] Mengnan Du, Xingming Wu, Weihai Chen, Jianhua Wang, " Fusing Region Contrast and Graph Regularization for Saliency Detection," in 27th Chinese Control and Decision Conference, 2015, pp. 5789-5794.

[17] Y. Fu, J. Cheng, Z. Li, and H. Lu, "Saliency cuts: An automatic approach to object segmentation," in Proc. IEEE Int. Conf. Pattern Recognit., 2008, pp. 1-4.
[18]H. J. Seo and P. Milanfar, "Nonparametric bottom-up saliency detection by self-resemblance," in Proc. IEEE Int. Conf. Comput. Vis. Pattern Recognit. Workshops, 2009, pp. 45-52.

[19] V. Gopalakrishnan, Y. Hu, and D. Rajan, "Salient region detection by modeling distributions of color and orientation," IEEE Trans.Multimedia, vol. 11, no. 5, pp. 892-905, Aug. 2009.

[20] L. Wang, J. Xue, N. Zheng, and G. Hua, "Automatic salient object extraction with contextual cue," in Proc. IEEE Int. Conf. Comput. Vis., Nov. 2011, pp. 105-112.

[21] V. Gopalakrishnan, Y. Hu, and D. Rajan, "Random walks on graphs for salient object detection in images," IEEE Trans. Image Process., vol. 19, no. 12, pp. 3232 3242, Dec. 2010.

[22] Y.-F. Ma and H.-J. Zhang, "Contrast-based image attention analysis by using fuzzy growing," in Proc. ACM Int. Conf. Multimedia, 2003, pp. 374-381. 